PAPER • OPEN ACCESS

Terahertz transitions in narrow-gap carbon nanotubes and graphene nanoribbons

To cite this article: V A Saroka et al 2018 J. Phys.: Conf. Ser. 1092012121

View the article online for updates and enhancements.

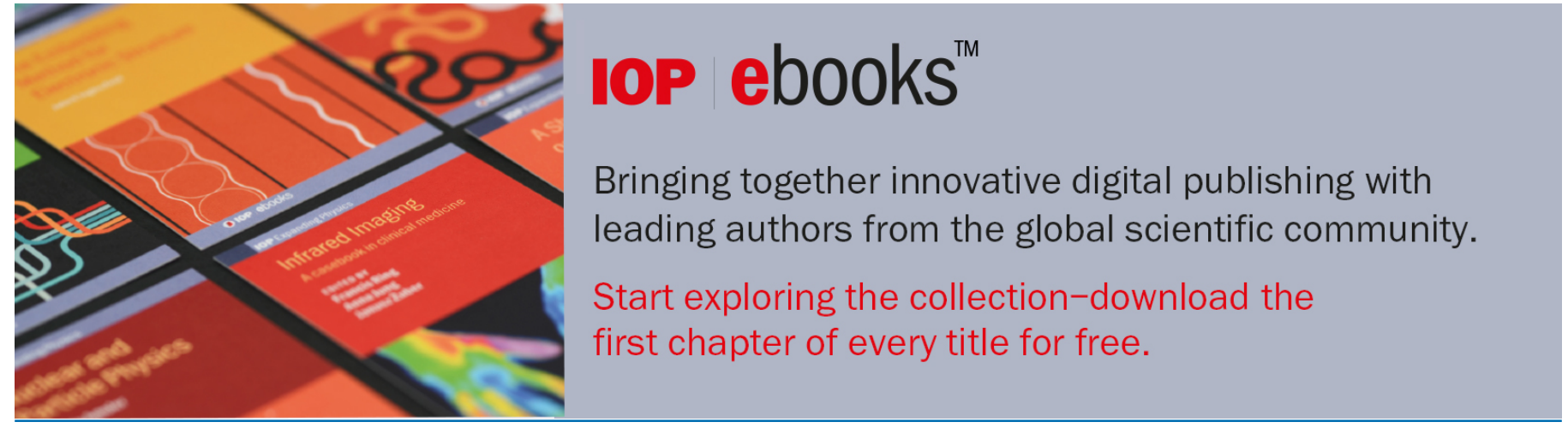

This content was downloaded from IP address 217.21.43.91 on 06/05/2021 at 10:27 


\title{
Terahertz transitions in narrow-gap carbon nanotubes and graphene nanoribbons
}

\author{
V A Saroka ${ }^{1,2}$, R R Hartmann ${ }^{3}$ and M E Portnoi ${ }^{1,4,5}$ \\ ${ }^{1}$ School of Physics, University of Exeter, Stocker Road, Exeter EX4 4QL, UK \\ ${ }^{2}$ Institute for Nuclear Problems, Belarusian State University, Minsk 220030, Belarus \\ ${ }^{3}$ Physics Department, De La Salle University, 2401 Taft Avenue, Manila, Philippines \\ ${ }^{4}$ ITMO University, St. Petersburg 197101, Russia
}

${ }^{5}$ Corresponding author: m.e.portnoi@exeter.ac.uk

\begin{abstract}
We show that the curvature effects in quasi-metallic carbon nanotubes and edge effects in narrow-gap graphene nanoribbons not only open band gaps in the THz range but also result in giant enhancement of the transition probabilities across these gaps. This makes these nanostructures perspective candidates for sources and detectors of $\mathrm{THz}$ radiation.
\end{abstract}

\section{Introduction}

Creating reliable, portable, tunable sources and detectors of terahertz $(\mathrm{THz})$ radiation is one of the most challenging tasks of contemporary applied physics. One of the recent trends in bridging the socalled $\mathrm{THz}$ gap is to use carbon-based nanostructures [1]. Several original schemes utilizing the unique electronic properties of carbon nanotubes (CNTs) and graphene for $\mathrm{THz}$ application were brought forward by our group [2-6]. These schemes include THz generation by hot electrons in quasimetallic CNTs, frequency multiplication in chiral-nanotube-based superlattices controlled by a transverse electric field, tunable $\mathrm{THz}$ radiation detection and optically-pumped emission in metallic CNTs in a strong magnetic field and using graphene p-n junctions for sub-wavelength polarizationsensitive $\mathrm{THz}$ detection. In the current presentation, we focus on direct interband dipole transitions in narrow-gap CNTs and graphene nanoribbons (GNRs).

\section{Narrow-gap carbon nanotubes and graphene nanoribbons}

The main conclusions of the reported work is that the curvature effects in quasi-metallic single-walled CNTs and edge effects in gapless graphene nanoribbons not only open bang gaps, which are typically in the $\mathrm{THz}$ range, but also result in giant enhancement of the probability of optical transitions across these gaps. Moreover, the matrix element of the velocity operator for these transitions has a universal value when the photon energy coincides with the band gap energy $E g$. Namely, the absolute value of this matrix element for band-edge transitions is equal to graphene's Fermi velocity $v_{F}$. Upon increasing the excitation energy the absolute value of the transition matrix element first rapidly decreases (for photon energies remaining in the $\mathrm{THz}$ range but exceeding $2 E g$ it is reduced by three orders of magnitude) and thereafter it starts to increase proportionally to the photon frequency. A similar effect occurs in an armchair CNT with a band gap opened and controlled by a magnetic field applied along the nanotube axis [4-6]. As can be seen from Fig. 1, there is a direct correspondence between armchair graphene nanoribbons (AGNRs) and single-walled zigzag CNTs. 

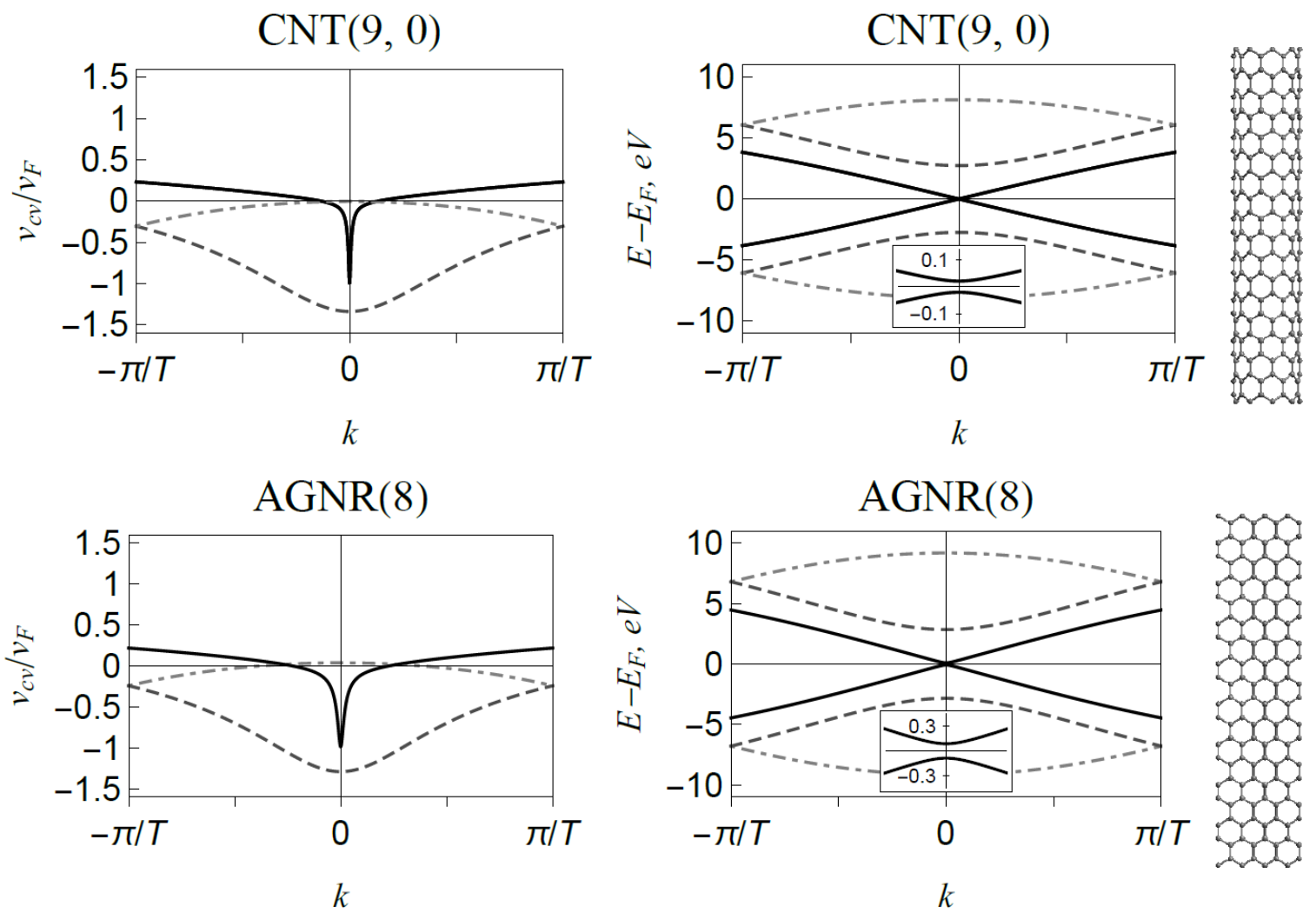

Figure 1. Energy spectra and interband transitions velocity matrix elements for CNT $(9,0)$ (upper panel) and AGNR (8) (lower panel) as a function of momentum in the first Brillouin zone.

The described sharp photon-energy dependence of the transition matrix element together with the van Hove singularity at the band gap edge of the considered quasi-one-dimensional systems make them promising candidates for active elements of coherent $\mathrm{THz}$ radiation emitters. The effect of Pauli blocking of low-energy interband transitions caused by residual doping can be suppressed by creating the population inversion using high-frequency (optical) excitation. Excitonic effects, which are known to dominate the optical properties of semiconductor CNTs, are of less importance in narrow-gap CNTs and ANGRs where the exciton binding energy is proportional to the bandgap [7] and dark excitonic states become irrelevant.

This work was supported by the EU H2020 RISE project CoExAN, FP7 ITN NOTEDEV and visitors program at ITMO.

\section{References}

[1] R. R. Hartmann, J. Kono, and M. E. Portnoi, Nanotechnology, 25, 322001 (2014).

[2] O. V. Kibis, D. G. W. Parfitt, and M. E. Portnoi, Phys. Rev. B 71, 035411 (2005).

[3] O. V. Kibis, M. Rosenau da Costa, and M. E. Portnoi, Nano Lett. 7, 3414 (2007).

[4] M. E. Portnoi, O. V. Kibis, M. Rosenau da Costa, Superlattices Microstruct. 43, 399 (2008).

[5] M.E. Portnoi, M. Rosenau da Costa, O.V.Kibis, I.A.Shelykh, Intern. J. Modern Phys. B 23, 2846 (2009).

[6] R.R.Hartmann and M.E.Portnoi, IOP Conf. Ser.: Mater. Sci. Eng. 79, 012014 (2015).

[7] R.R.Hartmann, I.A.Shelykh, and M.E.Portnoi, Phys. Rev. B 84, 035437 (2011). 